\title{
Computational screening of potential drugs against COVID-19 disease: the Neuropilin-1 receptor as molecular target
}

\author{
Hicham Charoute ${ }^{1,2}$ (D) Zouhair Elkarhat ${ }^{2} \cdot$ Lamiae Elkhattabi $^{2} \cdot$ Elmostafa El Fahime $^{3} \cdot$ \\ Naoual Oukkache ${ }^{4} \cdot$ Hassan Rouba $^{2} \cdot$ Abdelhamid Barakat $^{2}$
}

Received: 24 April 2021 / Accepted: 1 November 2021 / Published online: 21 January 2022

(C) The Author(s), under exclusive licence to Indian Virological Society 2021

\begin{abstract}
The transmembrane receptor Neuropilin-1 (NRP-1) was reported to serve as a host cell entry factor for the severe acute respiratory syndrome coronavirus 2 (SARS-CoV-2), the causal agent of COVID-19 disease. Therefore, molecular compounds interfering with SARSCoV-2 binding to NRP-1 seem to be potential candidates as new antiviral drugs. In this study, NRP-1 receptor was targeted using a library of 1167 compounds previously analyzed in COVID-19 related studies. The results show the effectiveness of Nafamostat, Y96, Selinexor, Ebastine and UGS, in binding to NRP-1 receptor, with docking scores lower than $-8.2 \mathrm{kcal} / \mathrm{mol}$. These molecules interact with NRP-1 receptor key residues, which makes them promising drugs to pursue further biological assays to explore their potential use in the treatment of COVID-19.
\end{abstract}

Keywords COVID-19 · SARS-CoV-2 · Neuropilin-1 . Molecular docking $\cdot$ Virtual screening $\cdot$ Antivirals

Hicham Charoute

hicham.charoute@pasteur.ma

1 Research Unit of Epidemiology, Biostatistics and Bioinformatics, 1, Place Louis Pasteur, Institut Pasteur du Maroc, 20360 Casablanca, Morocco

2 Laboratory of Genomics and Human Genetics, Institut Pasteur du Maroc, Casablanca, Morocco

3 Molecular Biology and Functional Genomics Platform, National Center for Scientific and Technical Research, Rabat, Morocco

4 Laboratory of Venoms and Toxins, Institut Pasteur du Maroc, Casablanca, Morocco

\section{Introduction}

Since the first identification of SARS-CoV-2, the drug discovery community is racing against time to find an active drug against this new virus. However, there is still no drug molecules that showed a therapeutic potential in COVID-19 disease treatment.

Besides severe acute respiratory syndrome coronavirus (SARS-CoV) and Middle-East respiratory syndrome (MERS-CoV), SARS-CoV-2 is the new member of Coronaviridae family and among coronaviruses that infect humans [30]. The genome of coronaviruses is composed of a large positive-sense and single-stranded RNA of approximately $30 \mathrm{~kb}$. It encodes four structural proteins; the spike $(\mathrm{S})$ protein, the nucleocapsid $(\mathrm{N})$ protein, the membrane (M) protein, and the envelope (E) protein, all necessary for the production of complete infectious virus particle [18]. Moreover, the SARS-CoV-2 genome comprises 16 functional non-structural proteins (nsp1 up to nsp16) implicated in the proteolytic cleavage and synthesis and processing of RNA [21].

Different SARS-CoV-2 proteins have attracted attention of scientists working on the development of new small molecules inhibitors using computer-aided drug design and discovery approaches. The spike (S) is the most important structural protein, it interacts with the Human ACE2 receptor (angiotensin 2 converting enzyme) as an entry point into cells $[8,14]$. The nsp5 non-structural protein, known to be the coronavirus main protease (Mpro). It plays a critical role in the life cycle of the coronavirus as its enzymatic activity cleaves polyproteins to produce functional proteins [1]. Among these functional proteins, the nsp12 (RNA-dependent RNA polymerase) plays an essential role in the replication and transcription of SARSCoV-2 RNA genome [6]. 
Instead of targeting viral proteins, some researchers have focused on the human receptors involved in the SARS-CoV-2 virus entry into host cells. The angiotensinconverting enzyme 2 (ACE2) receptor plays a principal role in the binding and fusion of the virus with the host cell membrane. During the cell entry of the viral particles, the SARS-CoV-2 spike (S) proteins are cleaved by Furin protease into receptor binding subunit $\mathrm{S} 1$ and membrane fusion subunit $\mathrm{S} 2$. The $\mathrm{S} 1$ subunit specifically binds into ACE2 receptor via its receptor-binding domain (RBD), while $\mathrm{S} 2$ subunit mediates the cell fusion after proteolytic activation by the transmembrane protease serine 2 (TMPRSS2) [11, 19].

Proteolytic cleavage of S1-S2 junction in the spike (S) protein by Furin generated a conserved C-terminus amino acid motif 682-RRAR-685[4]. This motif conforms to the C-end terminal rule (CendR) which is characterized by the following configuration $[\mathrm{R} / \mathrm{K}] \mathrm{XX}[\mathrm{R} / \mathrm{K}]$ (where $\mathrm{R}$ is arginine that can be substituted by lysine $(\mathrm{K})$ and $\mathrm{X}$ can be any amino acid)[12]. Serval studies showed that CendR peptides can interacts with the b1 domain of the Neuropilin-1 (NRP1) transmembrane receptor [7, 15, 22]. Recently, cumulative evidences coming from different studies has suggested that NRP-1 receptor may facilitate the entry of SARS-CoV-2 into the host cell, through the interaction between its $\mathrm{B} 1$ domain and the $\mathrm{S} 1$ subunit of the spike (S) glycoprotein [3, 4]. Thus, NRP-1 receptor may represent a potential therapeutic target for antiviral drugs against COVID-19 disease.

Computer aided drug discovery and design approaches such as molecular docking and virtual screening enables analyzing the affinity between a huge number of chemical compounds and a protein target in relatively short time. Thus, the use of these computational methods allows scientists to save time and reduce the cost of drug design workflows. The aim of this paper is to identify new inhibitors against NRP-1 receptor to inhibit the binding of the spike (S) protein and subsequent reduction of the viral infectivity of SARS-CoV-2.

\section{Materials and methods}

\section{Database of compounds}

To support research efforts in development of new treatments against the SARS-CoV-2 virus, the PubChem database provides a compiled list of information related to COVID-19 and SARS-CoV-2. This aggregated data helps PubChem users understand compounds have already been reported to have a potential effect against COVID-19.
Three-dimensional structure of 1167 compounds involved with COVID-19 related studies (vivo and in vitro studies, SARS-CoV-2 clinical trials, COVID19-related PDB structures) were downloaded from PubChem in SDF file format. In addition, the SDF files of two previously described inhibitors of NRP-1 receptor (EG00229 and EG01377) were also downloaded from PubChem. All these ligands were prepared and saved in PDBQT file format using OpenBel software.

\section{Preparation of receptor}

The crystal structure of the b1b2 domains from human Neuropilin-1 receptor (PDB ID: 2QQI) was downloaded from the protein databank. Co-crystallized ligand and water molecules were selected and removed using Chimera software. The preparation of receptor protein was done using the AutoDockTools 1.5.6 (ADT).

\section{Docking}

The AutoDock Vina software was used to carry out a virtual screening analysis against a library of compounds to finding novel and potent inhibitor of the Neuropilin-1 receptor. The docking grid box parameters were set to cover all key residues responsible for contacting the CendR motif (Y297, W301, T316, D320, S346, T349 and Y353). Thus, the grid box center was set to $X=19.836$, $\mathrm{Y}=29.418$ and $\mathrm{Z}=42.159$, and the dimensions of the box were set as $16 \times 16 \times 18 \AA$ with a spacing of $1 \AA$ between the grid's points. The best docking complexes having the minimal binding energy were considered for further analyzes using Discovery Studio software.

\section{In-silico drug-likeness analysis}

Drug-likeness analysis is an important step in the drug discovery workflow, which helps scientists to make a decision on whether a candidate drug can be safely administered to human body or not. Lipinski's rule of five evaluate the drug-likeness of a chemical molecule based on some key pharmacological properties (molecular weight, hydrogen bonding and lipophilicity). According to this rule, a chemical compound can be orally absorbed if it has no more than one violation of the following properties: molecular weight $\leq 500$ daltons, hydrogen bond acceptors $\leq 10$, hydrogen bond donors $\leq 5$ and octanol/water partition coefficient $\leq 5$. The drug-likeness analysis was performed using ADMETlab 2.0 web server [26]. The input SMILES strings of all compounds were obtained from PubChem database. 
Table 1 Docking scores and structures of top five compounds

$\begin{array}{ll}\text { Compound ID } & \text { Compound Name } \\ & \text { Nafamostat } \\ \text { CID154815735 } & \begin{array}{l}\text { 5-[(E)- } \\ \text { (hydroxyimino)methyl]- } \\ \text { 2-methyl-N-[(1R)-1- } \\ \text { (naphthalen-1- } \\ \text { yl)ethyl]benzamide } \\ \text { (Y96) } \\ \text { Selinexor } \\ \text { Cina score } \\ \text { (kcal/mol) }\end{array} \\ \text { CID71481097 } & \begin{array}{l}\text { EutoDock } \\ \text { Ebastine } \\ \text { yl)-2-(naphthalen-2- } \\ \text { yl)acetamide (UGS) }\end{array} \\ \text { CID3191 } & \end{array}$

\section{Ligand-binding pockets comparison}

The comparison of ligand-binding sites was performed using PocketAlign v1.1 algorithm [29] that provides a detailed alignment between binding pockets and best residue correspondences.

\section{Compound clustering}

Potential Neuropilin-1 protein inhibitors identified in this work were compared to those reported in two published studies. The first study proposed 10 compounds (N1 to N10) with high probability of interacting with Neuropilin-1 receptor based on molecular docking predictions. The second study, demonstrated using an enzyme linked immunosorbent assay (ELISA) that the compounds 2, 4, 5, 8, 9 and 17 can effectively inhibit Neuropilin-1.

The compounds dataset was clustered based on similarities between chemical structures using the K-Means classification algorithm implemented in ChemMine tools
[2]. Data were input into this Web-based cheminformatics tool using SMILES format.

\section{Results}

In the present study, a virtual screening analysis was performed using a database of 1167 compounds against the NRP1 receptor. The selection criteria of the best drug candidates was based on their Autodock Vina binding scores and (lower scores indicate stronger binding affinities) interactions with the active site key residues. The docking scores and interaction modes of the top five selected compounds are summarized in Table 1, Figs. 1 and 2 .

The docking results revealed that Nafamostat (Pubchem ID: CID4413) drug showed the highest binding affinity to the NTP1 protein with a binding energy of $-8.6 \mathrm{kcal} / \mathrm{mol}$. The Nafamostat is stabilized by four hydrogen bonds in which two interactions were observed with the active residues Asp320 and Tyr353. This compound forms also 


\section{A Nafamostat (CID4413)}

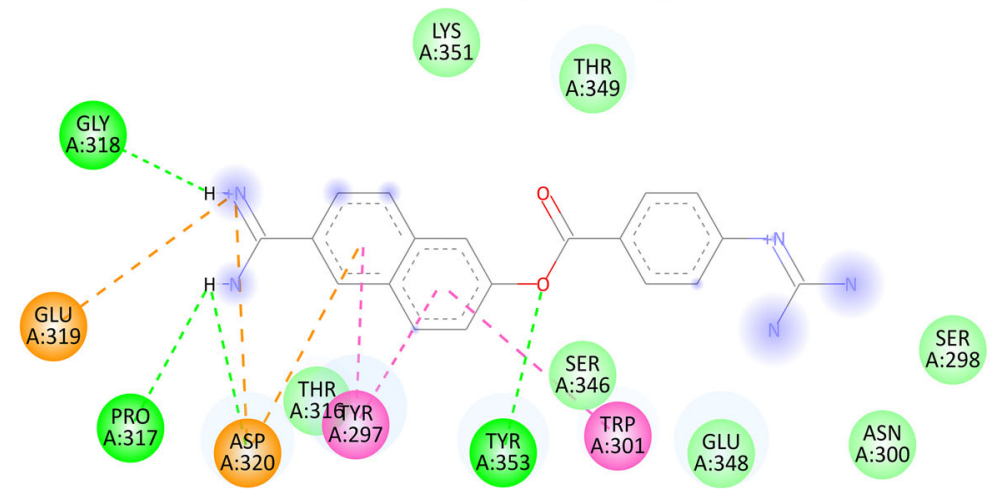

B Y96 (CID154815735)

C Selinexor (CID71481097)

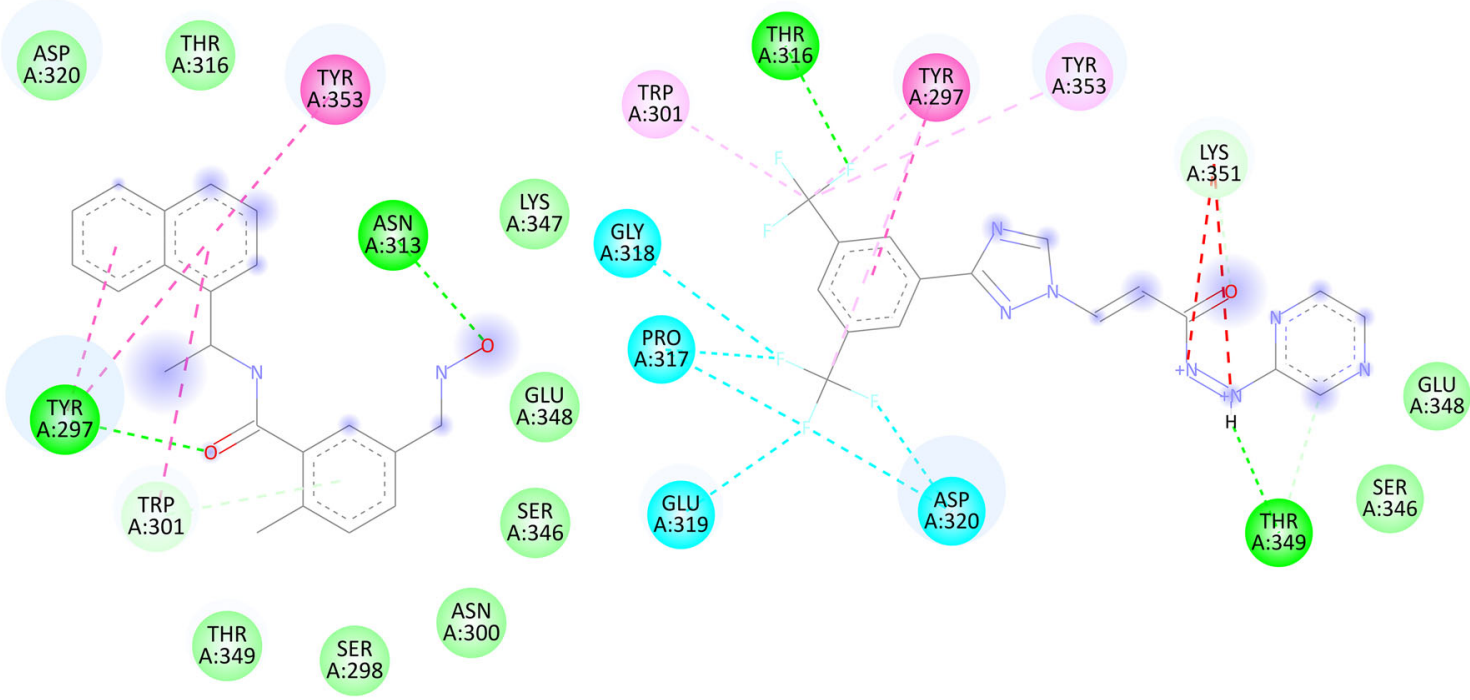

Interactions

\begin{tabular}{|l}
\hline van der Waals \\
Conventional Hydrogen Bond \\
Halogen (Fluorine) \\
Attractive Charge \\
Pi-Anion \\
Unfavorable Positive-Positive
\end{tabular}

Pi-Pi Stacked

Pi-Pi T-shaped

Pi-Alkyl

Carbon Hydrogen Bond

Pi-Donor Hydrogen Bond

Fig. 1 The best predicted binding poses of Nafamostat (a), Y96 (b) and Selinexor (c) compounds in the Neuropilin-1 active site

two hydrophobic interactions with key residues Tyr297 and Trp301 (Fig. 1a).

Y96 (CID154815735) and Selinexor (CID71481097) were both ranked at the second positions with a binding energy of $-8.4 \mathrm{kcal} / \mathrm{mol}$. Y96 formed two hydrogen bonds with Asn313 and the active residue Tyr297. In addition, three hydrophobic interactions were formed between Y96 and the key residues Tyr353, Trp301 and Tyr297. The contact mode showed that hydrogen bonds were formed between Selinexor and active residues Thr316 and Thr349. Furthermore, Trp301, Tyr297 and Tyr353 keys residues also interact with Selinexor through hydrophobic interactions (Fig. 1b, c).

Among the selected best hits, Ebastine (CID3191) and UGS (CID146037575) were ranked at the fourth and fifth positions with binding energies of -8.3 and $-8.2 \mathrm{kcal} /-$ mol, respectively. The Ebastine established two hydrophobic interactions with active amino acids Tyr353 and Trp301, and one hydrogen bond with Tyr353. UGS interact with active amino acids through two hydrogen 
A Ebastine (CID3191)

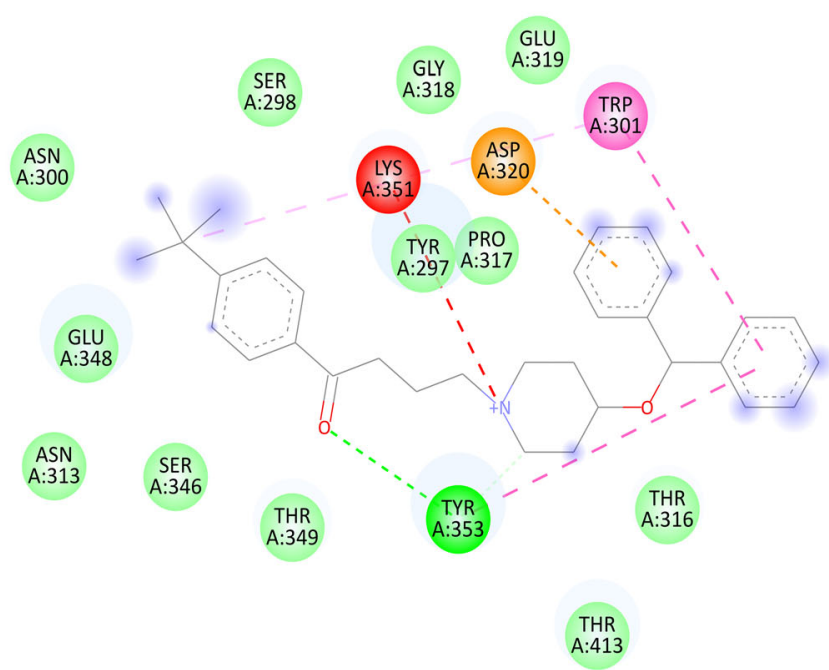

Interactions

van der Waals

Conventional Hydrogen Bond

Halogen (Fluorine)

Attractive Charge

Pi-Anion

Unfavorable Positive-Positive
B UGS (CID146037575)

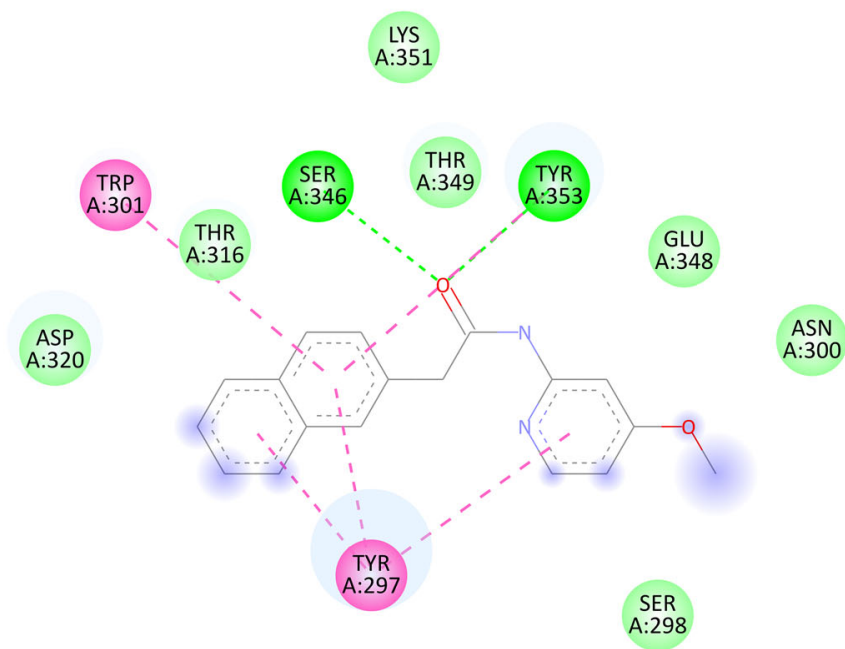

Fig. 2 The best predicted binding poses of Ebastine (a) and UGS (b) compounds in the Neuropilin-1 active site

bonds (Ser346 and Try353) and three hydrophobic interactions (Try353, Trp301 and Tyr297) (Fig. 2a, b).

All the top five compounds showed binding affinities better than the reference compounds EG00229 $(-6.6 \mathrm{kcal} / \mathrm{mol})$ and EG01377 $(7.1 \mathrm{kcal} / \mathrm{mol})$ (Table 2). The output of in-silico drug-likeness analysis is presented in Table 3. The five compounds are in concordance with Lipinski's rule. Two compounds violated only one criteria, Nafamostat has more than 5 hydrogen bond donors and Ebastine has a partition coefficient higher than 5. In contrast, both reference compounds EG00229 and EG01377 violated the Lipinski's rule.

\section{Discussion}

Structure-based drug design is a computer aided drug discovery approach, which use the three-dimensional structure of a target protein to identify potential active chemical compounds. During this pandemic situation of COVID-19, computational screening of large libraries of small molecules can accelerate the process of antiviral drug discovery and allow scientists to find the most promising inhibitors that specifically target SARS-CoV-2 cell entry pathway, virus RNA genome replication and maturation of viral particles. The study of SARS-CoV-2 cell entry mechanisms demonstrated that the Neuropilin-1 (NRP1) receptor can interact with the spike (S) protein and participate in the host cell entry mechanisms. Thus, this protein may be considerate as therapeutic target for the development of adjuvant drugs to interfere with SARS-CoV-2 cell entry.

In the current coronavirus outbreak, many in silico drug discovery studies were based on repurposing strategy to identified safe and effective medicines for COVID-19 treatment. This approach of scientific research is based on the use of drugs already approved for other diseases, to accelerate the response to health emergencies. In this context, we performed a molecular docking using 1167 compounds to interact we residues (Y297, W301, T316, D320, S346, T349 and Y353) implicated in the interaction between the spike (S) protein and NRP1 receptor. The screened compounds were retrieved from the PubChem database, which provides additional information on compounds that have been investigated in COVID-19 related studies. We found the molecular compounds Nafamostat, Y96, Selinexor, Ebastine and UGS to be the most suitable inhibitors for the NRP1 receptor. The molecular docking results showed that the binding energies of these 
Table 2 Docking scores of Neuropilin-1 inhibitors EG00229 and EG01377

\begin{tabular}{llll}
\hline Compound ID & Compound Name & 2D structure & $\begin{array}{c}\text { AutoDock } \\
\text { Vina score } \\
\text { (kcal/mol) }\end{array}$ \\
\hline CID44631827 & EG00229 & EG01377
\end{tabular}

Table 3 Drug likeness analysis

\begin{tabular}{|c|c|c|c|c|c|}
\hline Compound name & Molecular weight & Num. H-bond acceptors & Num. H-bond donors & $\log \mathrm{P}$ & Lipinski rule \\
\hline Nafamostat & 347.140 & 7 & 7 & 1.645 & Accepted \\
\hline Y96 & 332.150 & 4 & 2 & 4.108 & Accepted \\
\hline Selinexor & 443.090 & 8 & 2 & 3.749 & Accepted \\
\hline Ebastine & 469.300 & 3 & 0 & 6.397 & Accepted \\
\hline UGS & 292.120 & 4 & 1 & 3.136 & Accepted \\
\hline EG00229 & 497.060 & 12 & 7 & 0.630 & Rejected \\
\hline EG01377 & 586.170 & 12 & 9 & 0.821 & Rejected \\
\hline
\end{tabular}

$\log P \log$ of the octanol/water partition coefficient

ligands are lower than $-8.2 \mathrm{kcal} / \mathrm{mol}$, and the predicted protein-ligand interactions involved many key residues.

Nafamostat is a serine protease that is commonly used in Japan for the treatment of acute pancreatitis [5]. A previous study reported that Nafamostat exhibits a potential antiviral activity against the Middle East Respiratory Syndrome Coronavirus (MERS-CoV), by targeting the host protease TMPRSS2 that plays an essential role in the membrane fusion [28]. Recent reports suggested that Nafamostat can also inhibits the SARS-CoV-2 cell fusion and viral infection of human lung cells [9, 27]. We compared ligand binding pockets of NRP1 and TMPRSS2 proteins to identify structural and functional similarities that may justify the interaction with NAFAMOSTAT drug. PocketAlign software showed that the biding pockets of the two proteins share 8 similar residues (Fig. 3). Furthermore, a molecular docking analysis was conducted to study the interaction of the top 5 hits with the TMPRSS2 protein active site. All compounds showed good binding scores, ranging from -6.0 to $-7.5 \mathrm{kcal} / \mathrm{mol}$. These results suggest that this list of ligands could be considered as good inhibitors for NRP1 and TMPRSS2 proteins (Supplementary data, Table S1).

Literature research showed that Selinexor can be considered as drug candidates for the COVID-19 treatment. Clinical studies investigating the use of the exporting 1 protein (XPO1) inhibitor Selinexor as treatment of COVID-19 disease are in progress [23]. The cellular function of this protein is required for nucleocytoplasmic transport and it's inhibition by Selinexor may reduce SARS-CoV-2 virus replication.

Ebastine is an antihistaminic drug that has been approved for the treatment of allergic rhinitis. Repurposing studies showed encouraging results which potentiate Ebastine as good candidate of the COVID-19 treatment. In vitro screening of 30 approved medicines reveals that Ebastine displayed a strong inhibition of the SARS-CoV-2 main protease (Mpro) in infected cells [24]. In addition, a 


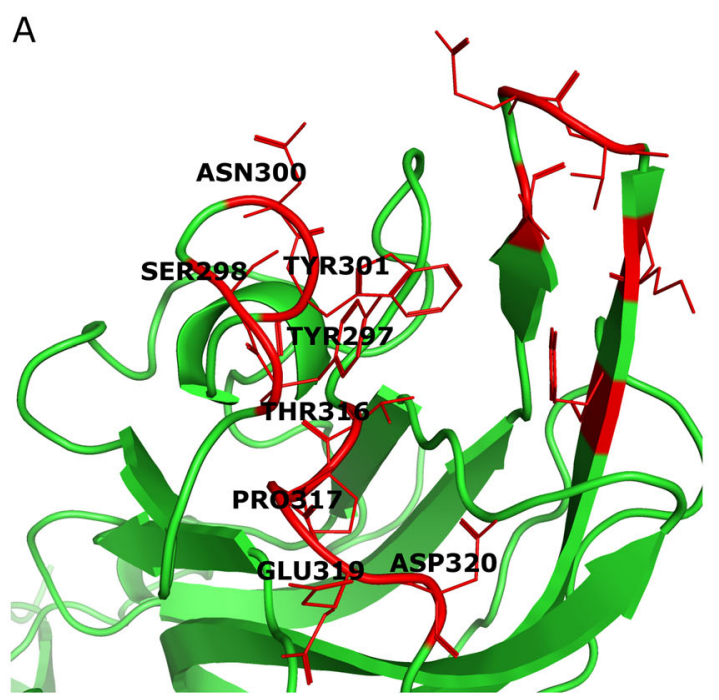

C

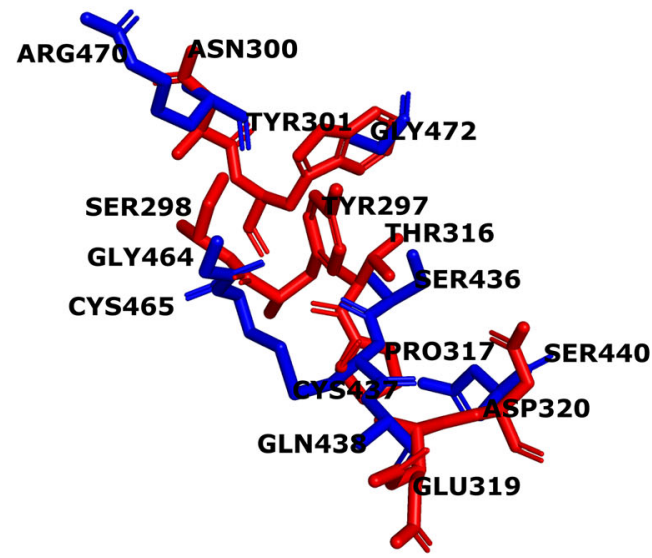

Fig. 3 Alignment of Nafamostat binding pockets. Binding pocket of NRP1 protein (a), Binding pocket of TMPRSS2 protein (b), similar amino acids (c) and distance between similar amino acids (d). All

preprint paper reported the ability of this drug in slowing the replication process of SARS-CoV-2 in human cells [17].

Both ligands Y96 and UGS were co-crystallized with two SARS-CoV-2 proteins, Papain-Like Protease (PDB ID $7 \mathrm{KOL}$ ) and Mpro main protease (PDB ID 5RGY), respectively. The inhibition of these enzymes, which plays an essential in cleavage and maturation of polyproteins, can disturbs the viral spread $[1,10,13,20]$.

The docking scores and drug-like physico-chemical properties of the top five small molecules were compared with the most well-known NRP-1 protein inhibitors; EG00229 and EG01377. The obtained results showed that all the analyzed compounds interact with NRP-1 more effectively than both reference compounds EG00229 and EG01377. Thus, this list of compounds represents an opportunity to develop more effective drugs against NRP-1 receptor.

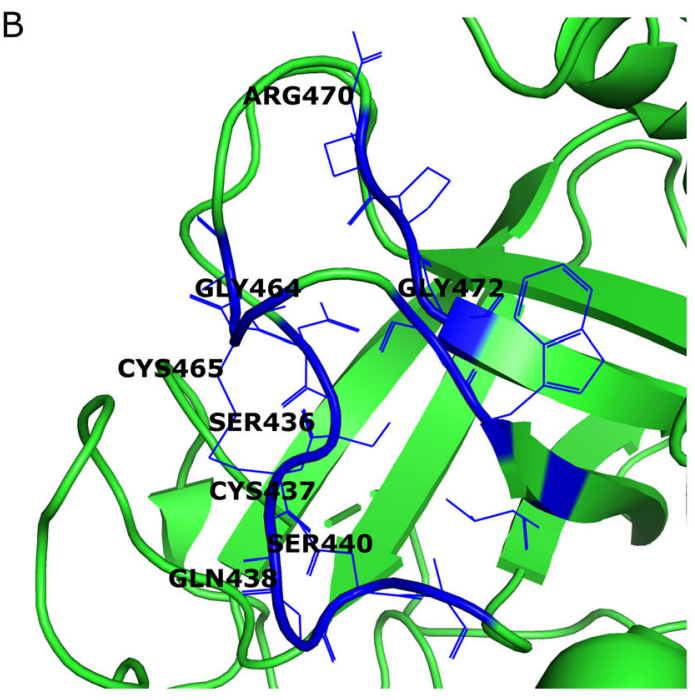

D

\begin{tabular}{ccc}
$\begin{array}{c}\text { Neuropilin-1 } \\
\text { PDB ID: 2qqi }\end{array}$ & $\begin{array}{c}\text { TMPRSS2 } \\
\text { PDB ID: } 7 \mathrm{meq}\end{array}$ & Disrance $(\AA$ \\
\hline TYR 297 & GLY 464 & 3.5 \\
SER 298 & CYS 465 & 3.1 \\
ASN 300 & ARG 470 & 2.2 \\
TRP 301 & GLY 472 & 5.3 \\
THR 316 & SER 436 & 1.5 \\
PRO 317 & CYS 437 & 1.8 \\
GLU 319 & GLN 438 & 1.3 \\
ASP 320 & ASP 440 & 1.3
\end{tabular}

amino acids located in the binding pocket of NRP1 and TMPRSS2 proteins are colored in red and blue, respectively

A structural comparison was conducted between the top 5 five molecules (Nafamostat, Y96, Selinexor, Ebastine and UGS) with those reported in two published studies [16, 25]. Based on the K-means algorithm, all chemical compounds were clustered into three main groups. The majority of top hits (Nafamostat, Y96, Selinexor and UGS) belongs to a group that includes two experimentally validated inhibitors (Compounds 4 and 5) (Fig. 4).

\section{Conclusion}

Until the writing of this paper, the COVID-19 disease is still representing a public health problem and several clinical studies are ongoing to identify effective antiviral drugs. Here, a structure-based docking to screen molecular compounds previously investigated in COVID-19 
Fig. 4 Structural classification of chemical compounds

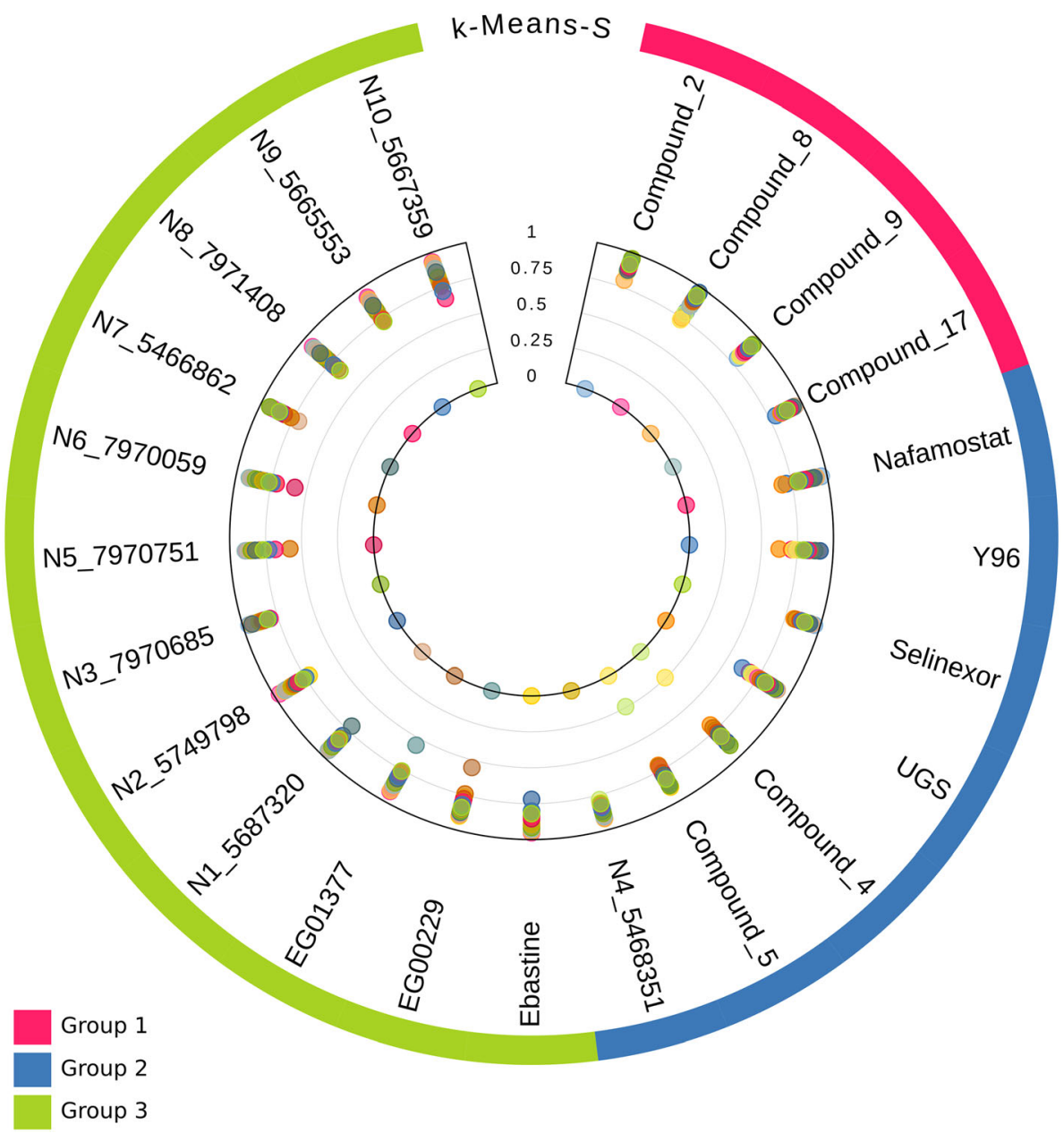

disease related studies. The obtained result implies that Nafamostat, Y96, Selinexor, Ebastine and UGS may emerge as good candidates for the inhibition of the interaction between the spike (S) protein and NRP1 receptor. These findings are promising and need further in vitro and in vivo testing of the identified compounds against the SARS-CoV-2 infection.

Supplementary Information The online version contains supplementary material available at https://doi.org/10.1007/s13337021-00751-x.

Acknowledgements Virtual screening was conducted on the HighPerformance Computing cluster of the National Center for Scientific and Technical Research (CNRST), Rabat, Morocco.

Funding This work was supported by the Hassan II Academy of Science and Technology as part of the research project entitled: "Innovative and Integrated Investigations for the development of diagnostic tests, screening tests and adapted treatments".

\section{Declarations}

Conflict of interest The authors declare that they have no conflict of interest.

\section{References}

1. Anand K, Ziebuhr J, Wadhwani P, Mesters JR, Hilgenfeld R. Coronavirus main proteinase (3CLpro) structure: basis for design of anti-SARS drugs. Science. 2003;300:1763-7.

2. Backman TWH, Cao Y, Girke T. ChemMine tools: an online service for analyzing and clustering small molecules. Nucleic Acids Res. 2011;39:W486-491.

3. Cantuti-Castelvetri L, Ojha R, Pedro LD, Djannatian M, Franz J, Kuivanen $\mathrm{S}$, et al. Neuropilin-1 facilitates SARS-CoV-2 cell entry and infectivity. Science. 2020;370:856-60.

4. Daly JL, Simonetti B, Klein K, Chen K-E, Williamson MK, Antón-Plágaro C, et al. Neuropilin-1 is a host factor for SARSCoV-2 infection. Sci Am Assoc Adv Sci. 2020;370:861-5.

5. Fuwa M, Kageyama M, Ohashi K, Sasaoka M, Sato R, Tanaka M, et al. Nafamostat and sepimostat identified as novel neuroprotective agents via NR2B N-methyl-D-aspartate receptor antagonism using a rat retinal excitotoxicity model. Sci Rep Nat Publ Group. 2019;9:20409

6. Gao Y, Yan L, Huang Y, Liu F, Zhao Y, Cao L, et al. Structure of the RNA-dependent RNA polymerase from COVID-19 virus. Science. 2020;368:779-82.

7. Guo H-F, Vander Kooi CW. Neuropilin functions as an essential cell surface receptor. J Biol Chem. 2015;290:29120-6.

8. Hoffmann M, Kleine-Weber H, Schroeder S, Krüger N, Herrler $\mathrm{T}$, Erichsen S, et al. SARS-CoV-2 cell entry depends on ACE2 
and TMPRSS 2 and is blocked by a clinically proven protease inhibitor. Cell. 2020;181:271-280.e8.

9. Hoffmann M, Schroeder S, Kleine-Weber H, Müller MA, Drosten C, Pöhlmann S. Nafamostat mesylate blocks activation of SARSCoV-2: new treatment option for COVID-19. Antimicrob Agents Chemother [Internet]. Am Soc Microbiol J. 2020 [cited 2021 Mar 26];64. Available from: https://aac.asm.org/content/64/6/e0075420

10. Jin Z, Du X, Xu Y, Deng Y, Liu M, Zhao Y, et al. Structure of M pro from COVID-19 virus and discovery of its inhibitors. Nat Publ Group. 2020;1-9

11. Kyrou I, Randeva HS, Spandidos DA, Karteris E. Not only ACE2 - the quest for additional host cell mediators of SARSCoV-2 infection: Neuropilin-1 (NRP1) as a novel SARS-CoV-2 host cell entry mediator implicated in COVID-19. Sig Transduct Target Ther Nat Publ Group. 2021;6:1-3.

12. Mayi BS, Leibowitz JA, Woods AT, Ammon KA, Liu AE, Raja A. The role of Neuropilin-1 in COVID-19. PLOS Pathog Public Lib Sci. 2021;17:e1009153.

13. Osipiuk J, Azizi S-A, Dvorkin S, Endres M, Jedrzejczak R, Jones $\mathrm{KA}$, et al. Structure of papain-like protease from SARS-CoV-2 and its complexes with non-covalent inhibitors. Nat Commun. 2021;12:743.

14. Ou X, Liu Y, Lei X, Li P, Mi D, Ren L, et al. Characterization of spike glycoprotein of SARS-CoV-2 on virus entry and its immune cross-reactivity with SARS-CoV. Nat Commun. 2020;11:1620.

15. Pang H-B, Braun GB, Friman T, Aza-Blanc P, Ruidiaz ME, Sugahara KN, et al. An endocytosis pathway initiated through neuropilin- 1 and regulated by nutrient availability. Nat Commun. 2014;5:4904.

16. Perez-Miller S, Patek M, Moutal A, Duran P, Cabel CR, Thorne $\mathrm{CA}$, et al. Novel compounds targeting neuropilin receptor 1 with potential to interfere with SARS-CoV-2 virus entry. ACS Chem Neurosci Am Chem Soc. 2021;12:1299-312.

17. Pickard A, Calverley BC, Chang J, Garva R, Lu Y, Kadler KE. Discovery of re-purposed drugs that slow SARS-CoV-2 replication in human cells. bioRxiv [Internet]. Cold Spring Harbor Laboratory Preprints; 2021 [cited 2021 Mar 31]; Available from: https://www.ncbi.nlm.nih.gov/pmc/articles/PMC7872348/

18. Schoeman D, Fielding BC. Coronavirus envelope protein: current knowledge. Virol J. 2019;16:69.

19. Shang J, Wan Y, Luo C, Ye G, Geng Q, Auerbach A, et al. Cell entry mechanisms of SARS-CoV-2. Proc Natl Acad Sci. 2020;117:11727-34.
20. Shin D, Mukherjee R, Grewe D, Bojkova D, Baek K, Bhattacharya A, et al. Papain-like protease regulates SARS-CoV-2 viral spread and innate immunity. Nature Publ Group. 2020;587:657-62.

21. Snijder EJ, Decroly E, Ziebuhr J. The nonstructural proteins directing coronavirus RNA synthesis and processing. Adv Virus Res. 2016;96:59-126.

22. Teesalu T, Sugahara KN, Kotamraju VR, Ruoslahti E. C-end rule peptides mediate neuropilin-1-dependent cell, vascular, and tissue penetration. Proc Natl Acad Sci USA. 2009;106:16157-62.

23. Uddin MdH, Zonder JA, Azmi AS. Exportin 1 inhibition as antiviral therapy. Drug Discov Today. 2020;25:1775-81

24. Vatansever EC, Yang KS, Drelich AK, Kratch KC, Cho C-C, Kempaiah KR, et al. Bepridil is potent against SARS-CoV-2 in vitro. Proc Natl Acad Sci USA [Internet]. 2021 [cited 2021 Mar 31];118. Available from: https://www.ncbi.nlm.nih.gov/ pmc/articles/PMC7958448/

25. Vique-Sánchez JL. Potential inhibitors interacting in Neuropilin1 to develop an adjuvant drug against COVID-19, by molecular docking. Bioorg Med Chem. 2021;33:116040

26. Xiong G, Wu Z, Yi J, Fu L, Yang Z, Hsieh C, et al. ADMETlab 2.0: an integrated online platform for accurate and comprehensive predictions of ADMET properties. Nucleic Acids Res. 2021;49:W5-14.

27. Yamamoto M, Kiso M, Sakai-Tagawa Y, Iwatsuki-Horimoto K, Imai $\mathrm{M}$, Takeda $\mathrm{M}$, et al. The anticoagulant nafamostat potently inhibits SARS-CoV-2 S protein-mediated fusion in a cell fusion assay system and viral infection in vitro in a cell-type-dependent manner. Viruses. 2020;12.

28. Yamamoto M, Matsuyama S, Li X, Takeda M, Kawaguchi Y, Inoue $\mathrm{J}$, et al. Identification of nafamostat as a potent inhibitor of middle east respiratory syndrome coronavirus s protein-mediated membrane fusion using the split-protein-based cell-cell fusion assay. Antimicrob Agents Chemother. 2016;60:6532-9.

29. Yeturu K, Chandra N. PocketAlign A novel algorithm for aligning binding sites in protein structures. J Chem Inf Model Am Chem Soc. 2011;51:1725-36

30. Zhu N, Zhang D, Wang W, Li X, Yang B, Song J, et al. A Novel coronavirus from patients with pneumonia in China, 2019. N Engl J Med. 2020;382:727-33.

Publisher's Note Springer Nature remains neutral with regard to jurisdictional claims in published maps and institutional affiliations. 